\title{
Hipercementosis Apicales y No Apicales en Raíces Dentarias Humanas
}

\author{
Apical and Non-Apical Hypercementosis in Human Dental Root
}

*Alicia Kohli; **Stella M. Pezzotto \& ** Leonor Poletto

KOHLI, A.; PEZZOTTO, S. M. \& POLETTO, L. Hipercementosis apicales y no apicales en raíces dentarias humanas. Int. J. Morphol., 29(4):1263-1267, 2011.

RESUMEN: El cemento secundario del ápice radicular se deposita en forma lenta y continua durante toda la vida útil del diente, siendo su función compensar el desgaste producido por la masticación. En raras ocasiones podemos hallar en la raíz dental, a posteriori de una extracción dentaria, excesos de cementos redondeados u ovalados, bien delimitados y duros al tacto, ubicados en un sector radicular diferente al ápice. Nuestro objetivo fue comparar las características físicas y radiológicas de raíces dentales normales con aquéllas que presentan este tipo de formaciones que alteran la anatomía radicular. Fueron incluidos 20 dientes adultos con raíces normales y 20 con raíces donde se visualizaron estos depósitos. Las variables investigadas fueron edad, sexo, patologías generales y periodontales previas, tipo de diente extraído, ubicación en el arco dental, motivo de extracción, características de consistencia, color, aspecto y textura de todas las raíces. La imagen radiológica fue crucial para la inclusión de dientes con exceso de cemento. Se recolectó un total de 40 dientes adultos, cuya edad promedio fue de 61años (16-79), 42.5\% masculinos y 57.5\% femeninos. Patología general presentó el 37.5\% y periodontal el $55 \%$, los dientes mas extraídos fueron premolares superiores del lado derecho $15 \%$. La caries fue causa principal de extracción $72.5 \%$. En ambos grupos las raíces fueron de consistencia dura y textura lisa. El aspecto nacarado fue de $70 \%$ y $60 \%, 10 s$ colores claros $45 \%$ y $75 \%$, respectivamente. En las radiografías se observó que el cemento no se diferenció de la dentina en el $40 \%$ y $85 \%$ de cada grupo $(\mathrm{p}=0.004)$. Los adultos pueden desarrollar anomalías de cemento ubicadas en otro sector del ápice si bien las características físicas de la raíz se consideran dentro de parámetros normales.

PALABRAS CLAVE: Cemento dental; Hipercementosis; Perlas de cemento.

\section{INTRODUCCION}

El depósito de cemento secundario en el ápice radicular, más común de observar, es lento y sostenido a lo largo de la vida útil del diente. Su función es compensar el desgaste oclusal manteniendo a las piezas dentales en contacto determinando el plano de oclusión (Abramovich, 1999). Según su ubicación se denomina: 1 . Apical: al que se encuentra, mas frecuentemente, en el ápice y tercio apical de la raíz Su aspecto histológico puede ser de capas de cemento dispuestas en láminas ordenadas o irregulares con abundantes cementocitos, líneas de reposo oblicuas o incurvadas con respecto del eje mayor de la raíz, con vasos incluidos o bien tener el aspecto de los sistemas de Haver del tejido óseo compacto (Cabrini, 1980). Se considera que factores locales como falta de antagonista, traumatismo e inflamación periodontal crónica influyen en su aparición la que también puede ser ocasionada por la enfermedad de Paget (Thoma, 1984). Este tipo de hipercementosis puede hallar- se en uno o varios dientes, siendo mas común en premolares, en personas adultas jóvenes y mayores; como la vitalidad pulpar no está comprometida suele ser un hallazgo casual por radiografía o extracción dentaria (Monahan, 1994). Otra es la 2. Cementosis difusa: que es más difícil de observar, se puede presentar con formas ovoide o esférica y estar ubicada en otra zona de la raíz, por ejemplo en tercios medio y cervical. Esta provoca una deformación definida, histológicamente semejante a la apical la cual ha sido relacionada con la enfermedad de Paget (Cabrini).

$\mathrm{Al}$ extraer una pieza dental resulta sorpresivo hallar excesos de cemento con forma de perlas bien delimitados y duros al tacto, ubicados en sectores diferentes al ápice radicular. Ante este hallazgo, el clínico se plantea el interrogante de estar frente a una hipercementosis apical o bien ante el desarrollo de alguna variedad de cementoma, con 
los cuales se debería realizar el diagnóstico diferencial (Gorlin \& Meskin, 1963.; Larsson et al., 2003; Grinspan, 1983). Estas evaginaciones de cemento también podrían estar relacionadas con procesos inflamatorios crónicos como fuera demostrado en una niña con erupción precoz de un premolar inferior permanente debido a la destrucción por caries del molar deciduo (Aguiló \& Gandía, 1995). En un estudio realizado sobre los cuerpos de esclavos africanos de Barbados se las relacionó con la progresión de la enfermedad periodontal y la mala nutrición crónica (Corruccini et al., 1987). La inflamación crónica y la hipercementosis rodeando un implante de titanio se hallaron en un animal investigado (Guarnieri et al., 2002). En una joven de 17 años se encontró hipercementosis en la cercanía del cuello anatómico de molares superiores con reabsorción radicular idiopática e inflamación (Schätzle et al., 2005).

El objetivo de este trabajo es comparar las características clínicas de las hipercementosis apicales con los depósitos de cemento no apical, relacionándolos con causas locales como caries o enfermedad periodontal crónica que hayan motivado la extracción de la pieza dental, incluyendo el padecimiento de alguna enfermedad general.

\section{MATERIAL Y MÉTODO}

Se utilizaron 20 dientes permanentes extraídos, superiores e inferiores, de ambos sexos, de forma radicular considerada normal y otras 20 piezas en cuyas raíces se observaron depósitos de cemento en forma redondeada o de perlas que se encontraron ubicadas alejadas del ápice radicular.

A las personas donantes se les preguntó por su edad, padecimiento de patologías previas (diabetes, enfermedad de Paget, hiperpituitarismo), incluida la enfermedad periodontal crónica y el motivo de la extracción de la pieza dental. Se procedió a fotografiar cada una de las piezas normales obtenidas y en el sitio de la evaginación a las que presentaron los depósitos de cemento. Se tomaron radiografías de las piezas normales y las que presentaron perlas para analizar características de radiopacidad o radiolucidez. Se analizaron las características físicas del cemento de todas las raíces entre ellas color desde el blanco, la gama de amarillos crema en tonos muy pálido, pálido, medio, intenso, oscuro tipo mostaza y por último la mezcla de colores resultante de la exposición bucal así como la consistencia o dureza al tacto. El brillo de las raíces en brillante tipo pulido, nacarado y opaco. La textura fue lisa cuando al tacto los dedos resbalaron sobre ellas como en superficie pulida, rugosa cuando al tocarlas se halló dificultad para el deslizamiento y otra opción fue la mezcla de texturas lisa y rugosa.

En las raíces con perlas se evaluó su consistencia la que debió ser dura para incluirlas en el estudio. El volumen producido por la protuberancia fue clasificado en leve, moderado y prominente. Se registró el número de perlas, las que según la forma fueron clasificadas en redondeadas, ovaladas o irregulares. Si su prominencia se diferenciaba del cemento vecino se las agrupó como bien delimitadas, caso contrario como poco delimitadas. Se midieron con un calibre clasificando su tamaño en $\mathrm{mm}$. El color fue igual o diferente al cemento radicular. Su aspecto y textura fueron evaluados de acuerdo con las características consideradas para todas las raíces.

Se calculó edad promedio y desvío estándar. Para las variables categóricas se calcularon proporciones, las que se compararon por medio de pruebas de Fisher con un nivel de significación del 5\%.

\section{RESULTADOS}

Patología general previa tuvo el 37,5\%; diabetes 25\% e hipertensión arterial 22,5\%. En los diabéticos 5\% la tuvo como único padecimiento, $12,5 \%$ la presentó asociada con hipertensión y 7,5\% con la enfermedad de Paget; en los hipertensos en $10 \%$ fue única patología. Se recolectó un total de 40 dientes adultos, cuya edad promedio fue de 61 años (16-79), $42,5 \%$ masculinos y $57,5 \%$ femeninos. Veinte fueron dientes con raíces consideradas normales y 20 con depósitos anormales de cemento visualizados después de realizada la extracción (Fig. 1).

La caries fue causa principal de extracción (72,5\%), seguido de movilidad dental $(27,5 \%)$. Los grupos dentarios se formaron con dientes anteriores $(37,5 \%)$, molares (35\%) y premolares $(27,5 \%)$. Por su lugar en el arco dentario predominaron los dientes superiores del lado derecho (37,5\%), seguido de inferiores derechos (25\%), de superiores izquierdos $(22,5 \%)$ y por último de inferiores izquierdos $(17,5 \%)$.

En las raíces con perlas los grupos dentarios se formaron con dientes anteriores (10\%), posteriores $(55 \%)$ y premolares (35\%). Por su lugar en el arco predominaron los dientes postero-superiores derechos (40\%), postero-inferiores derechos (20\%), postero-superiores izquierdos $(20 \%)$ y postero-inferiores izquierdos $(10 \%)$. No se halló ninguna pieza de los grupos antero superior derecho e izquierdo pero se contó con una pieza dentaria derecha y otra izquierda del grupo antero inferior (Tabla I). 

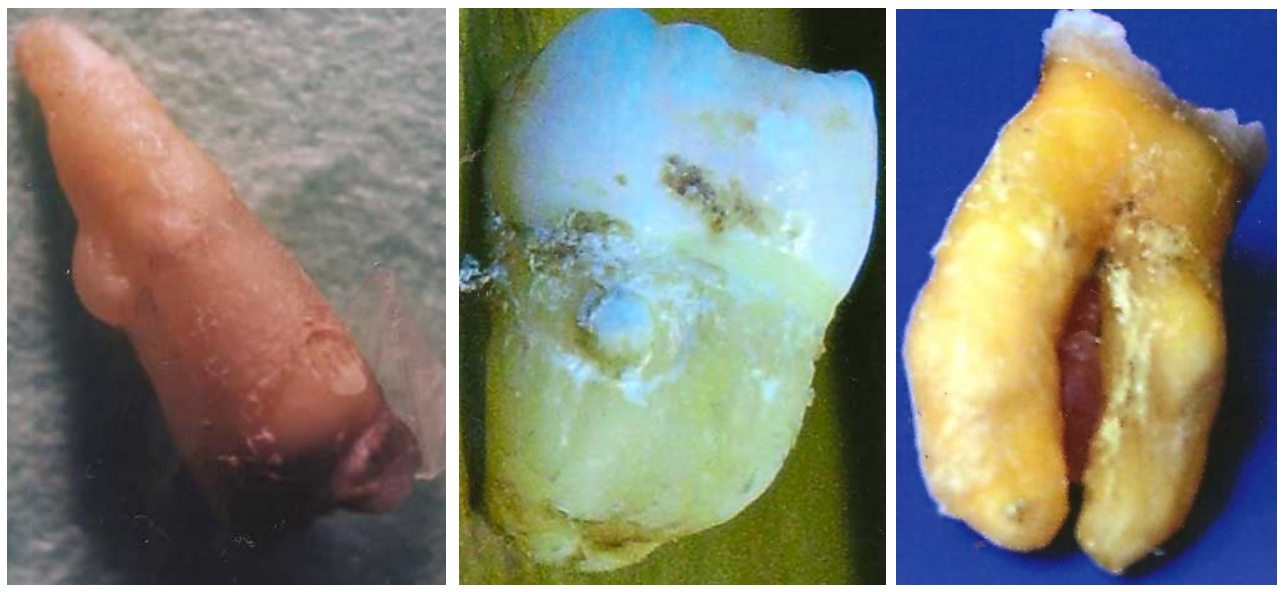

Fig. 1. Depósitos anormales de cemento en raíces dentarias.

Tabla I. Grupo dental y pertenencia al arco dentario en raíces con perlas.

\begin{tabular}{|c|c|c|c|c|c|c|c|c|c|c|c|c|}
\hline \multirow{2}{*}{$\begin{array}{l}\text { Grupo dental derecha e } \\
\text { izquierda }\end{array}$} & \multicolumn{2}{|c|}{ M D } & \multicolumn{2}{|c|}{ PM D } & \multicolumn{2}{|c|}{ I D y C D } & \multicolumn{2}{|c|}{ I I y C I } & \multicolumn{2}{|c|}{ PM I } & \multicolumn{2}{|c|}{ M I } \\
\hline & $\mathbf{n}$ & $\%$ & $\mathbf{n}$ & $\%$ & n & $\%$ & n & $\%$ & $\mathbf{n}$ & $\%$ & $\mathbf{n}$ & $\%$ \\
\hline Arco dental superior & 4 & 20 & 4 & 20 & & & & & 1 & 5 & 3 & 15 \\
\hline Arco dental inferior & 3 & 15 & 1 & 5 & 1 & 5 & 1 & 5 & 1 & 5 & 1 & 5 \\
\hline Total & 7 & 35 & 5 & 25 & 1 & 5 & 1 & 5 & 2 & 10 & 4 & 20 \\
\hline
\end{tabular}

M D: Molares derechos; PM D: Premolares derechos; I D y C D Incisivos y caninos derechos; I I y C I: Incisivos y caninos izquierdos; PM I: Premolares izquierdos; M I: Molares izquierdos.

Del análisis de propiedades físicas del total de raíces su consistencia fue dura. Predominaron los colores claros (85\%) variando desde casi blanco hasta amarillo oscuro. El aspecto nacarado de todas las raíces fue mayoría (65\%), seguido de brillante y opaco. La textura fue lisa en mayor pro- porción $(82,5 \%)$, y también rugosa y mezcla de ambas. La consistencia de las perlas fue dura en todos los casos; fueron de color blanco $(60 \%)$ y del mismo color que la raíz $(40 \%)$; predominó el aspecto nacarado $(75 \%)$ y su textura fue lisa en $90 \%$ (Fig. 2).

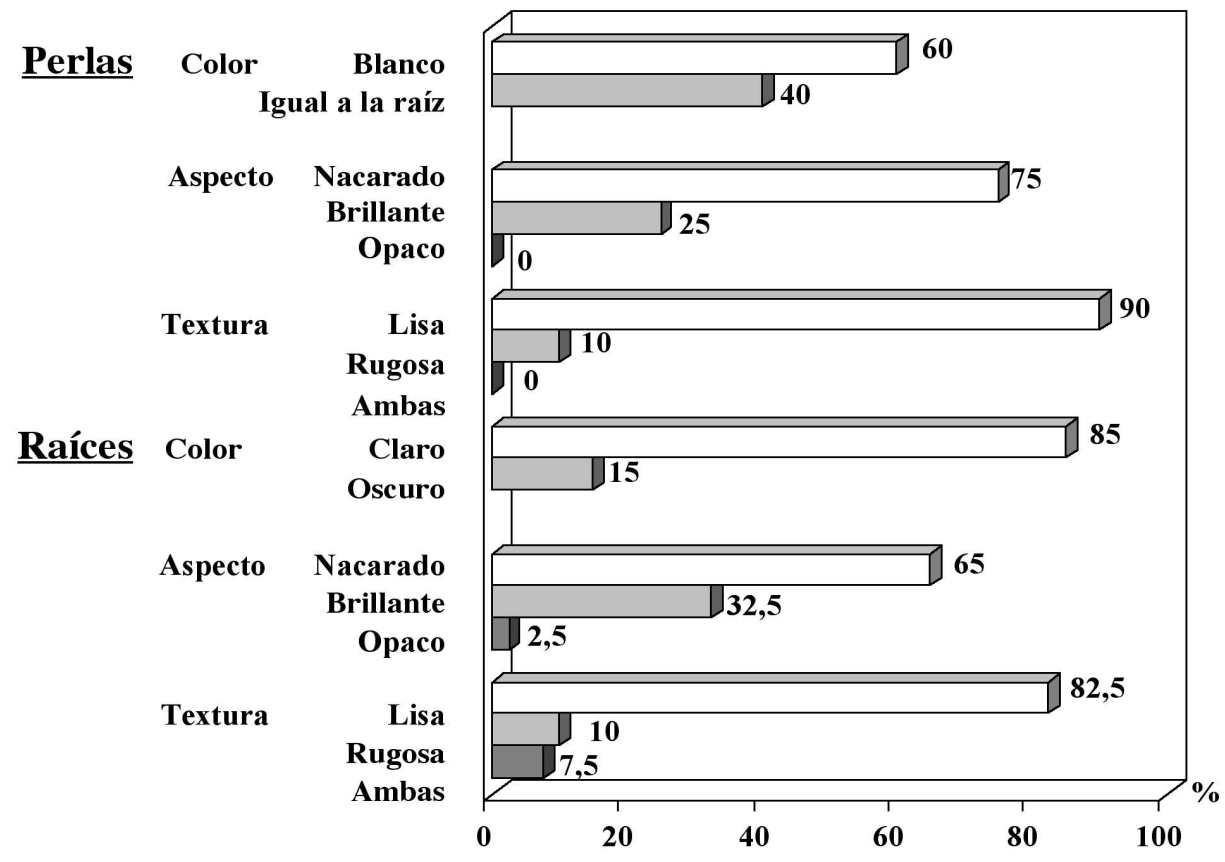

Fig. 2. Comparación de características físicas en raíces y perlas. 
El tamaño de su elevación fue clasificado en leve $45 \%$, moderado $15 \%$ y prominente $40 \%$. Estuvieron bien delimitadas del tejido radicular $90 \%$, las grandes midieron más de $3 \mathrm{~mm} 70 \%$, las medianas $3 \mathrm{~mm} 20 \%$ y las pequeñas menos de $3 \mathrm{~mm} 10 \%$. La imagen radiográfica, esencial para definir la inclusión de la perla, confirmó la proporción de leves, prominentes y moderadas obtenida al ser clasificadas en forma macroscópica y milimétrica. En las perlas no se diferenció la radio opacidad de cemento y dentina en un $85 \%$ y se diferenciaron en el $60 \%$ de las raíces normales $(\mathrm{p}=0,004)$. Su número varió de una en el 55\% (11/20), dos 30\% (6/20) y tres o más 15\% (3/ 20). Predominó la forma redondeada $70 \%$ sobre la ovalada $20 \%$ y la irregular 10\% (Fig. 3).

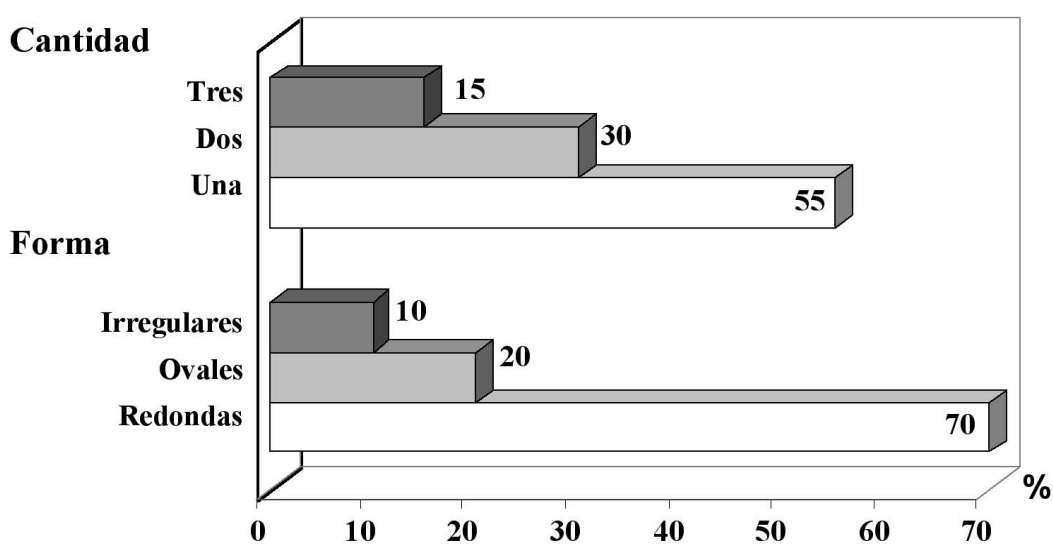

Fig. 3. Distribución de la forma y número de perlas.

\section{DISCUSION}

Las perlas fueron un hallazgo clínico posterior a una extracción dentaria. Debido al número escaso de estas observaciones se hizo necesario comparar las características generales de las mismas con posibles patologías relacionadas y así poder diferenciarlas. En un estudio de hipercementosis, se comprobó que éstas son más comunes en personas mayores, que los dientes afectados son uno o varios y generalmente son premolares (Monahan). En nuestro estudio, la población estuvo compuesta por personas adultas mayores pero el grupo dentario afectado incluyó premolares y molares. Según dicha investigación el depósito de cemento generalmente es bilateral, sin originar síntomas clínicos. Pero en nuestro grupo de hipercementosis las perlas no se presentaron de manera bilateral y su hallazgo fue casual en dientes aislados y con vitalidad pulpar comprometida. Se describió un tipo de hipercementosis ubicada en el ápice y tercio apical de la raíz con forma ovoide o esferoidal y otro exceso de cemento ubicado en la fusión de raíces y dilaceraciones a los que denominó hipercementosis difusa la que fue relacionada con la enfermedad de Paget (Cabrini). Las perlas de nuestro estudio fueron deformaciones cementarias definidas redondas u ovales coincidiendo con lo descrito por Cabrini, pero el hecho de encontrarlas en todos los tercios radiculares lejos del ápice las hace diferentes. También se halló en nuestro estudio que algunos pacientes, cuyos dientes presentaron perlas, padecían la enfermedad de Paget coincidiendo con las descripciones de otros investigadores (Aldred \& Cooke, 1989; Sashima et al., 1990). Dag`ıstan et al. (2007) reportaron un caso de hiperpituitarismo, en el cual las coronas dentales mantuvieron el tamaño normal pero las raíces de los dientes se alargaron acompañando el crecimiento del maxilar por medio de una hipercementosis apical, dicha enfermedad no se encontró en nuestros pacientes.

Se describe a los cementomas como más frecuentes en mandíbula (Ikeshima, 1995) patología no hallada en nuestros pacientes, en quienes las perlas se encontraron en ambos maxilares y desconocemos el factor causal de su predominio en dientes del lado derecho. La displasia fibrosa periapical fue descripta como frecuente en piezas dentarias antero-inferiores de mujeres con edad promedio de 39 años (Thoma); en nuestro estudio predominaron dientes del grupo medio y posterior en pacientes cuya edad promedio fue de 61 años. El cementoblastoma benigno fue diagnosticado en pacientes más jóvenes que los nuestros; no obstante, nuestras hipercementosis comparten con dicha patología la característica de ser un hallazgo poco frecuente y por consiguiente de la necesidad de realizar un diagnóstico diferencial (Napier Souza et al., 2004). En una investigación sobre pérdida de hueso alveolar en ratas con periodontitis crónica, se comprobó que ésta aumentaba con el paso del tiempo y a la vez se producía hipercementosis mesial en las raíces afectadas (Sashima et al). Coincidentemente, en nuestros pacientes con caries avanzada y movilidad dental, por procesos inflamatorios crónicos, se le sumaría el efecto de la edad mayor. Enfermedades previas padecidas por algunos pacientes, como ser diabetes que suele originar abscesos periodontales a repetición con inflamación gingival y movilidad dentaria con el transcurso del tiempo (Grinspan) reproducirían las condiciones para la aparición de las perlas (Sashima M). Además, en algunos pacientes, la diabetes se encontró relacionada a otra enfermedad que favorece el depósito de cemento como lo es el Paget (Aldred \& Cooke). Frente a la reducida información sobre trabajos similares sugerimos profundizar en este tema. Sería útil que el odontólogo general examinara la raíz dentaria luego de una extracción conociendo la infrecuencia de este hallazgo. 
KOHLI, A.; PEzzotTo, S. M. \& POLETTO, L. Apical and non-apical hypercementosis in human dental root. Int. J. Morphol., 29(4):1263-1267, 2011.

SUMMARY: Secondary cementum is deposited in a root apical third throughout the lifespan of a tooth to compensate occlusal erosion, keeping dental pieces in occlusion contact. Pearls are more difficult to spot, found by X-rays or after extraction. Their ovoid or spherical aspect is well outlined, hard to touch, and located in different thirds of the root. Our objective was to compare physical and radiological dental normal roots characteristics with those presenting this type of formations that alter radicular anatomy. We used 20 normal roots and 20 pearl shapes. Variables compared: age, sex, previous general pathologies and periodontals; extracted tooth type, dental arch location, extraction cause, consistency characteristics, color, aspect and texture of all roots and pearls. Radiological image was crucial for incorporation of teeth with cementum excess. Proportions between normal pieces and pearls were calculated with Fisher's Exact Test, with 5\% significance level. Average age was 61years (16-79), and male proportion was $42.5 \%$ and $57.5 \%$ female. General pathology presented $37.5 \%$ and periodontal $55 \%$, more extracted teeth were right side upper premolars $15 \%$. Caries were extraction chief motive $72.5 \%$. Roots were of hard consistency and smooth texture in both groups; nacreous aspect was $65 \%$, clear colors $75 \%$. Pearl consistency was hard in all of them; white color $60 \%$ and root similar color $40 \%$; nacreous aspect $75 \%$ and smooth texture $90 \%$. In radiographs was observed that cementum did not differ from dentine in $85 \%$ of pearls and in $60 \%$ of normal roots ( $\mathrm{p}=0.004$ ). Adults can develop cementum anomalies located in another apex sector although the root physical characteristics are considered inside normal parameters.

KEY WORDS: Dental cementum; Hypercementosis; Cementum pearls.

\section{REFERENCIAS BIBLIOGRÁFICAS}

Abramovich, A. Histología y Embriología dentaria. $2^{\circ}$ ed. Buenos Aires, Editorial Panamericana, 1999.

Aguiló, L. \& Gandía, J. Evaginación radicular. Rev. Act. Odontoestomat. Esp., 55:57-60, 1995.

Aldred, M. J. \& Cooke, B. E. Paget's disease of bone with involvement of the dental pulp. J. Oral Pathol. Med., 18(3):184-5, 1989.

Cabrini, R. Anatomía Patológica Bucal. $1^{\circ}$ ed. Buenos Aires, Editorial Mundi, 1980.

Corruccini, R. S.; Jacobi, K. P.; Handler, J. S. \& Aufderheide, A. C. Implications of tooth root hypercementosis in a Barbados slave skeletal collection. Am. J. Phys. Anthropol., 74(2):17984, 1987.

Dag ^ustan, S.; Go“"regen, M. \& Ogॅuzhan, A. Acromegaly: A case report. Internet Journal of Dental Science, 4(2), 2007. Available in: http://www.ispub.com/ostia/index.php?xmlFilePath= journals/ijds/vol4n2/acro.xml

Gorlin, R. J. \& Meskin, L. H. Odontogenic tumors in man and animals; pathologic classification and clinical behavior: a review. Ann. N. Y. Acad. Sci., 108:722-71, 1963.

Guarnieri, R.; Giardino, L.; Crespi, R. \& Romagnoli, R. Cementum formation around a titanium implant: a case report. Int. J. Oral Maxillofac. Implants, 17(5):729-32, 2002.

Grinspan, D. Semiología, patología, clínica y terapéutica de la mucosa bucal. Buenos Aires, Panamericana, 1983.
Ikeshima, A. Metrical differential diagnosis based on location (Part $2)$. Differential diagnosis among various benign tumors. $J$. Nihon Univ. Sch. Dent., 37(2):77-84, 1995.

Larsson, A.; Swartz, K. \& Heikinheimo, K. A case of multiple AOTlike jawbone lesions in a young patient--a new odontogenic entity? J. Oral Pathol. Med., 32(1):55-62, 2003.

Monahan, R. Periapical and localized radiopacities. Dent. Clin. North Am., 38(1):113-36, 1994.

Napier Souza, L.; Monteiro Lima Júnior, S.; Garcia Santos Pimenta, F. J.; Rodrigues Antunes Souza, A. C. \& Santiago Gomez, R. Atypical hypercementosis versus cementoblastoma. Dentomaxillofac. Radiol., 33(4):267-70, 2004.

Sashima, M.; Satoh, M. \& Suzuki, A. Alveolar bone loss of senescence-accelerated mouse (SAM). J. Dent. Res., 69(1):82$6,1990$.

Schätzle, M.; Tanner, S. D. \& Bosshardt, D. D. Progressive, generalized, apical idiopathic root resorption and hypercementosis. J. Periodontol., 76(11):2002-11, 2005.

Thoma, K. H. Patología Oral. $1^{\text {o }}$ ed. Barcelona, Salvat, 1984.

Dirección para correspondencia:

Alicia Kohli

Pasaje Rizzuto 3473

Rosario 2000, Santa Fe

ARGENTINA

Email: Aliciakohli2009@hotmail.com

Recibido : 16-03-2011

Aceptado: 14-07-2011 